01

\title{
Исследование быстродействия поверхностно-ионизационных детекторов
}

\author{
(C) А.Ш. Раджабов, С.С. Исхакова, Д.Т. Усманов \\ Институт ионно-плазменных и лазерных технологий им. У.А. Арифова, АН Республики Узбекистан, \\ 100125 Ташкент, Узбекистан \\ e-mail: a.radjabov0217@gmail.com
}

Поступило в Редакцию 28 мая 20211 г.

В окончательной редакции 28 июня 2021 r.

Принято к публикации 1 июля 2021 г.

Представлены результаты исследований, полученные методом термодесорбционной спектрометрии, направленных для изучения зависимости быстродействия поверхностного ионизационного детектора от температуры $\left(T_{E}\right)$ эмиттера. Для этого детектором регистрировались спектры испарения героина, папаверина и морфина, полученные в разных скоростях термопрограммированного нагрева веществ. Для изучения зависимости быстродействия детекторов от $T_{E}$ эмиттера данные спектры получены при разных $T_{E}$ эмиттера. Установлено, что с увеличением $T_{E}$ эмиттера быстродействия детектора увеличиваются. Выявлено, что это связано с увеличением (при увеличении $T_{E}$ ) скоростей гетерогенных процессов в адсорбционном слое эмиттера. Разработана математическая модель данных зависимостей, основанный на поверхностной ионизации молекул в нестационарных условиях.

Ключевые слова: эмиттер, термодесорбционная спектрометрия, поверхностно-ионизационный детектор, гетерогенные процессы.

DOI: 10.21883/JTF.2021.12.51753.164-21

\section{Введение}

Необходимость решения многих внелабораторных практических задач экологии, криминалистики, судебной медицины, токсикологии и наркологии [1-5] требует разработки относительно простых и недорогих, но селективных и высокочувствительных методов экспрессного физико-химического анализа для идентификации и определения концентрации микроколичеств примесей различных веществ в газовых смесях, экстрактах биопроб, продуктов питания и объектах окружающей среды [6-11]. Одним из таких методов, который может удовлетворять данным требованиям, является термодесорбционная (ТД) спектрометрия с поверхностноионизационным (ПИ) детектированием органических молекул [12-16]. ПИ детектор является селективным ионизационным детектором, работоспособным в условиях атмосферы воздуха [17-21], что является одним из важных особенностей ТД спектрометров, построенных на их основе. Работа ПИ детекторов основана на явлении ПИ органических молекул, содержащих гетероатом азота, находящего в функциональной связи с различными радикалами (алкильными, фенильными и др.) $[16,22]$. ПИ детекторы имеют высокую чувствительность и селективность к таким соединениям. Важность обнаружения следовых количеств данных веществ обусловлено тем, что они относятся к классу активных органических веществ, таких как наркотики, психотропные препараты, пестициды и другие токсичные классы веществ [23-28]. Поэтому развитие ПИ газоаналитического приборостроения позволяет решить ряд задач внелабораторной аналитической химии по анализу этих классов веществ.

Как известно, важнейшими параметрами детекторов являются их чувствительность, селективность, линейно динамический диапазон (ЛДД), стабильность и быстродействие [17,29-34]. На данный момент существует большое количество научно-практических работ, посвященных выявлению закономерностей ПИ и исследованию физико-химических свойств органических и биоорганических соединений [22-28], поиску эффективных и эмиссионно-стабильных ПИ эмиттеров для детекторов [35-39], определению чувствительности и ЛДД ПИ детекторов к веществам [17-21,2528], разработке методов обнаружения и анализа веществ в различных биологических образцах на основе ПИ детекторов [40-47]. Несмотря на это, отсутствуют сведения о закономерностях быстродействия ПИ детекторов.

Быстродействие является основным динамическим параметром детектора, характеризующим его способность быстро реагировать на резкое изменение концентрации вещества в потоке газа-носителя, проходящего через детектор, и является фактором, влияющим на интенсивность, форму и разделимость получаемых спектров. Быстродействие детектора зависит от физико-химических свойств ионизатора (или от физикохимических процессов в области ионизаций), от инерционности системы измерения сигнала (постоянная време- 

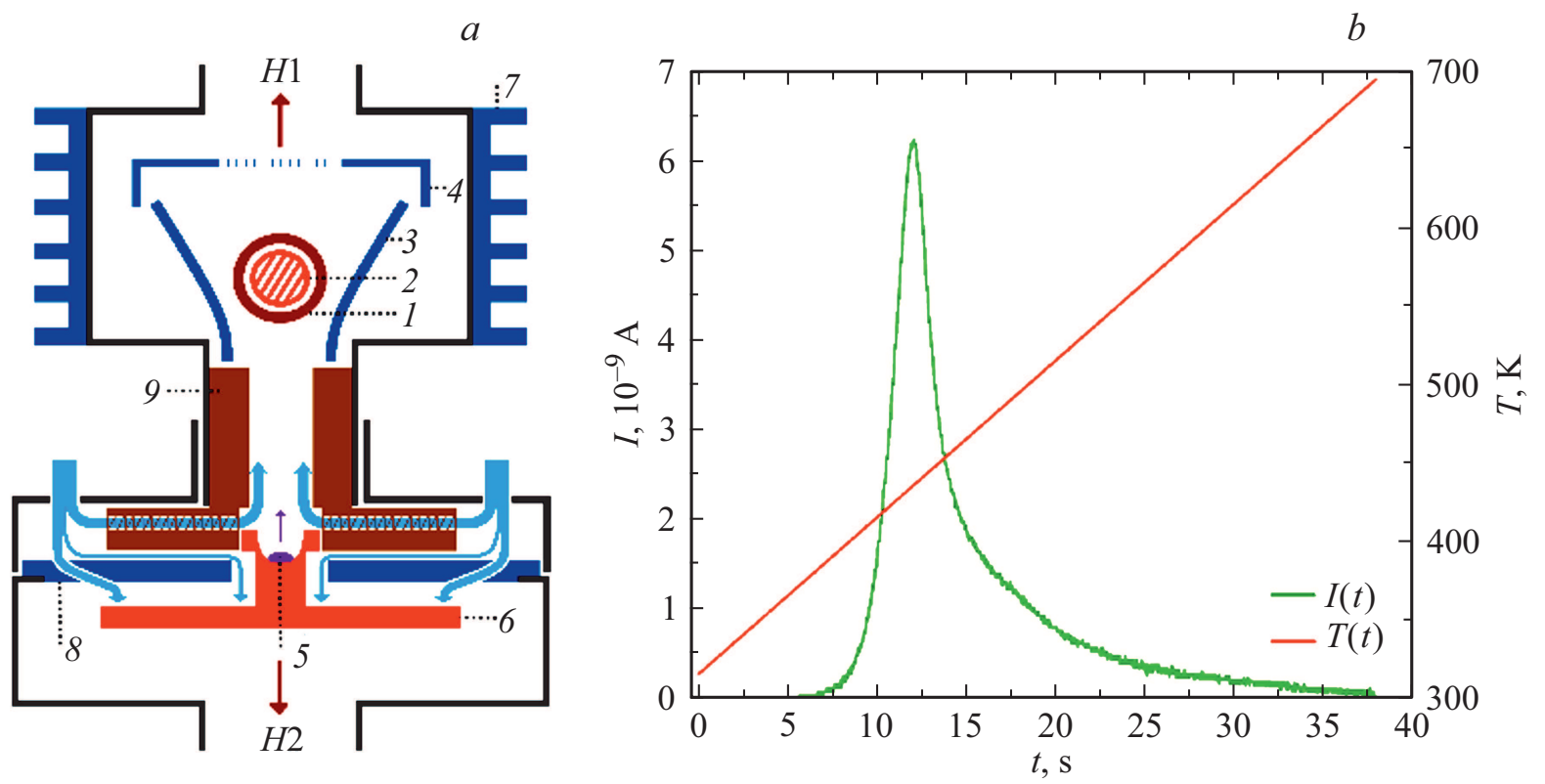

Рис. 1. $a-$ схема поверхностно-ионизационного детектора с испарителем, $b$ - принцип регистрации тока ионов и развертки температуры испарителя.

ни усилителя), скорости потока газ-носителя, объема и конструкции детектора [17,28-33].

Из закономерностей процессов ПИ известно [22,48-53], что, когда молекулы адсорбируются на поверхности эмиттера, часть из них подвергаются гетерогенным химическим реакциям по $i$-му каналу со скоростью $K_{i}^{d}=G_{i} \exp \left[-E_{i}^{d} / k T_{E}\right]$, из-за чего они преобразуются в $i$ вида ионизируемых частиц (например, $(\mathrm{M}-\mathrm{H}),(\mathrm{M}-\mathrm{R})$ и т.д.), которые десорбируются c эмиттера в нейтральном или ионном состоянии с соответствующими скоростями $K_{i}^{0}=C_{i} \exp \left[-E_{i}^{0} / k T_{E}\right]$ и $K_{i}^{+}=D_{i} \exp \left[-E_{i}^{+} / k T_{E}\right]$. Остальная часть адсорбированных молекул десорбируется, не подвергаясь химическим реакциям, со скоростью $K_{M}=C_{M} \exp \left[-E_{M} / k T_{E}\right]$, где $T_{E}$ - температура эмиттера; $E_{i}, E_{M}$ и $E_{i}$ - энергия активации соответствующих процессов; $C_{M}, G_{i}$ и $C_{i}-$ предэкспоненциальный множитель. Скорость данных процессов и их зависимость от $T_{E}$ может влиять на быстродействие детекторов.

В связи с этим целью настоящей работы является исследование зависимости быстродействия ПИ детекторов от $T_{E}$ эмиттера, что способствует развитию ПИ детекторов в аналитических областях.

\section{1. Экспериментальная техника и метод исследования}

Экспериментальное исследование производилось на лабораторном стенде, построенном на базе стандартного ПИ детектора и системы испарения веществ ТД спектрометров серии „Искович“ [12-15]. В соответствии с целью и методами исследования детектор и система испарения веществ модернизировались, их схема представлена в рис. $1, a$. Она состоит из анода-эмиттера 1 , нагреваемого косвенным накалом - нихромовой нитью 2; вытягивающего электрода 3 ; коллектора 4 в виде сетки с проницаемостью 50\%; вещества 5, наносимого на поверхность испарителя 6; насоса $H 1$, прокачивающего воздух-носитель испаряемых молекул веществ к детектору; системы воздушного охлаждения с алюминиевым радиатором 7, предназначенным для стабилизации температуры ионного источника. При прокачивание испаренных молекул веществ к детектору, также прокачивается горячий поток воздуха, выделяемый в системе испарения веществ, которой может привести к увеличению температуры эмиттера и источника ионов. Для предотвращения этого действия система испарения веществ оснащалась экраном 8 из нержавеющий стали, дополнительным насосом $H 2$ для прокачивания горячего воздуха из испарителя по отдельному каналу, а детектор оснащался металлокерамическим концентрическим переходником 9. Переходник имеет два входных канала. По первому прокачивается воздух-носитель, по второму испаряемые молекулы веществ. Стрелками обозначена линия воздушного потока.

Быстродействие детектора изучалось относительно хроматографически чистых (не менее 99\%) морфина, папаверина и героина. Хроматографически чистый папаверин фирмы „Alltech“(США), а морфин был получен в Управлении ООН по контролю за наркотиками (Вена, Австрия). Героин в микроколичестве был получен в Главном бюро судебно-медицинской экспертизы Республики Узбекистан. В качестве эмиттера ис- 

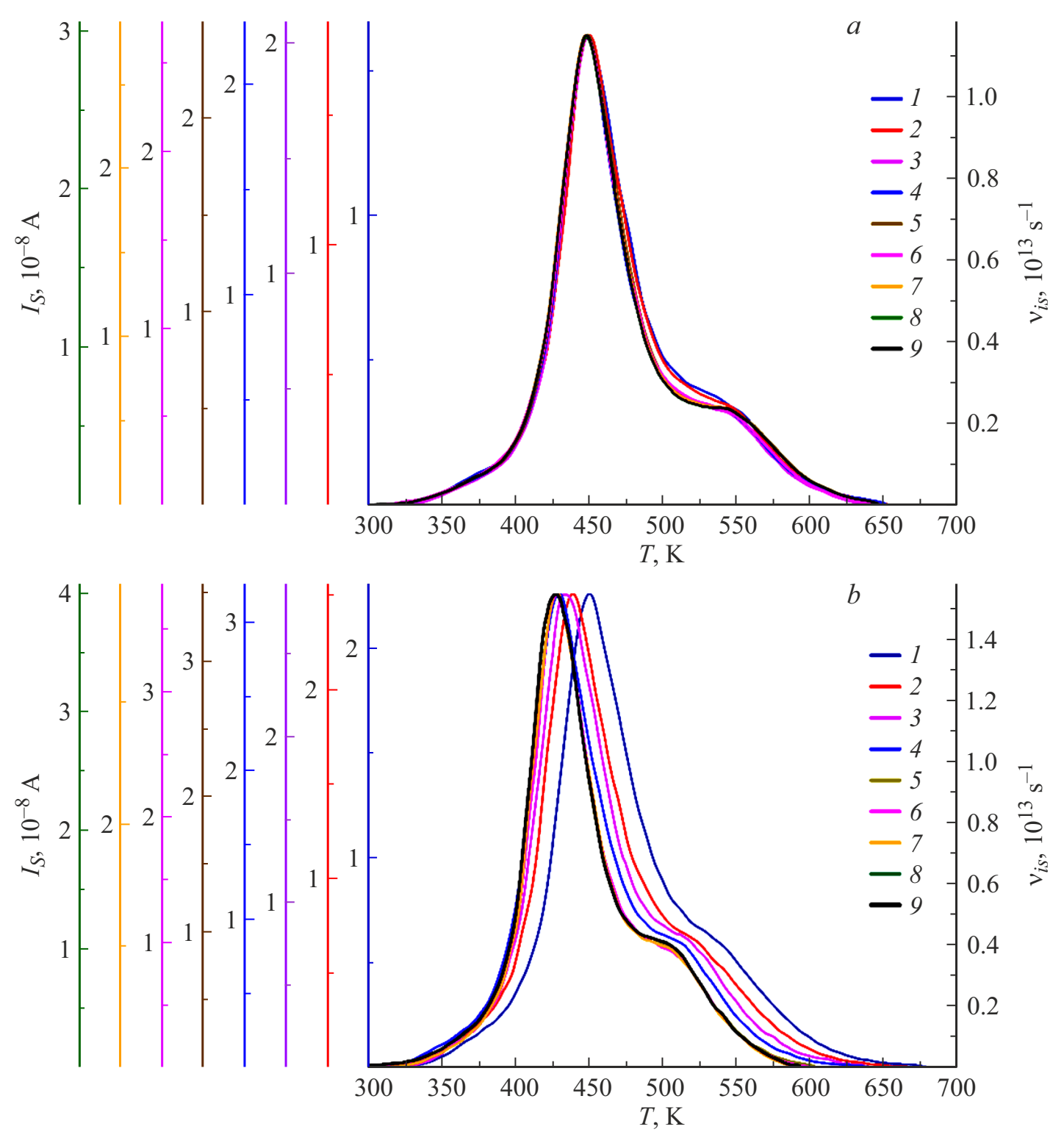

Рис. 2. тДПИ спектры героина: $a-$ при $7 ; b-14.2^{\circ} \mathrm{C} / \mathrm{s}$. Значение $T_{E}$, К: $1-639,2-649,3-659,4-668,5-678$, $6-685,7-692,8-699,9-$ поток молекул.

пользовался окисленный молибден, легированный иридием [35,36], выполненный в виде полого цилиндра длиной $12 \mathrm{~mm}$ и диаметром $2.5 \mathrm{~mm}$. $T_{E}$ эмиттера измерялась с помощью хромель-алюминиевой термопары, припаянной к $1 / 4$ его длины. Испаритель представляет собой графитизированную металлическую чашку диаметром $3 \mathrm{~mm}$ и высотой $1.5 \mathrm{~mm}$, температура которой также измерялась с помощью хромель-алюминиевой термопары. Его нагрев $T(t)$ осуществлялся относительно температуры $T_{0}=300 \pm 0.5 \mathrm{~K}$ с линейной скоростью $\omega$. Ток ионов $I_{s}(T)$ записывался синхронно с $T(t)$ (рис. $1, b)$ посредством цифрового осциллографа UTD2102 CEX (с программным обеспечением DSO Monitor Controller V1.8 и Wave Analysis фирмы UNI-Trend).
В настоящей работе для изучения зависимости быстродействия детектора от $T_{E}$ эмиттера были получены серии спектров при различных значениях $T_{E}$. Для изучения быстродействия детектора при разных скоростях изменения $d v_{i s} / d T$ потока молекул, поступающих на детектор, данная серия спектров получена при различных значениях скорости развертки $\omega$ температуры испарителя.

\section{2. Результаты и обсуждение}

На рис. 2 представлены термодесорбционные поверхностно-ионизационные (ТДПИ) спектры героина, полученные при разных температурах эмиттера $T_{E}$, а также спектр испарения героина $v_{i s}(T)$, рассчитанный 


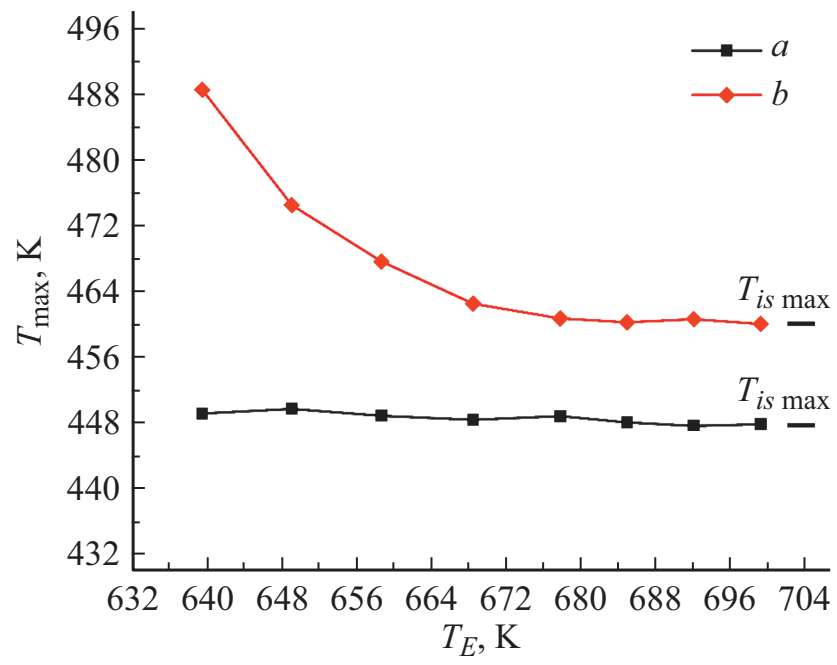

Рис. 3. Зависимость $T_{\max }$ от $T_{E}: a-$ при $7 ; b-$ при $14.2^{\circ} \mathrm{C} / \mathrm{s}$.

исходя из условий квазистационарности (методика вычисления $v_{i s}$ описана в конце разд. 2). Серия спектров получена при скорости температуры 7 (a) и $14.2^{\circ} \mathrm{C} / \mathrm{s}(b)$. Как видно из рис. $2, a$, с уменьшением температуры $T_{E}$ эмиттера интенсивность спектра уменьшается. Формы спектра зависимости $I_{S}(T)$ повторяли закономерность формы спектра испарения $v_{i s}(T)$. Температура максимума спектра осталась постоянной $T_{\max }=$ const, не зависела от $T_{E}$ и была равна температуре $T_{i s} \max$ максимума потока молекул $v_{i s} \max$ поступающих к детектору, т.е. $T_{\max }=T_{i s \max }=$ const (рис. 3,a). А при скорости $14.2^{\circ} \mathrm{C} / \mathrm{s}$ возникло два случая. В области температуры $T_{E}=678-699 \mathrm{~K}$ формы спектров $I_{S}(T)$ повторяли закономерность $v_{i s}(T)$ (рис. $2, b$ ). Температура $T_{\max }$ приняла постоянное значение $T_{\max }=$ const (рис. $3, b$ ). Однако в области $T_{E}=639-668 \mathrm{~K}$ закономерность $I_{S}(T)$ смещалась относительно закономерности $v_{i s}(T)$, а $T_{\max }$ зависела от $T_{E}$.

Схожие эффекты наблюдались также при исследовании папаверина. На рис. 4 представлены ТДПИ спектры и спектры испарения папаверина при разных $T_{E}$ эмиттера. На рис. 5 представлены графики зависимости $T_{\max }$ от $T_{E}$. Серии этих спектров, также получены при $7(a)$ и $14.2^{\circ} \mathrm{C} / \mathrm{s}(b)$. При $7^{\circ} \mathrm{C} / \mathrm{s}$ форма спектров $I_{s}(T)$ повторяла закономерность $v_{i s}(T)$, а $T_{\max }$ не зависела от $T_{E}$. Однако в отличие от спектров героина при $14.2^{\circ} \mathrm{C} / \mathrm{s}$ заметные смещения $I_{s}(T)$ от $v_{i s}(T)$ и $T_{\max }$ от $T_{i s \max }$ наблюдались при 639 и $649 \mathrm{~K} T_{E}$ эмиттера. Это говорит о том, что закономерность смещения $I_{s}(T)$ от $v_{i s}(T)$ и $T_{\max }$ от $T_{i s \max }$, возникшая при $14.2^{\circ} \mathrm{C} / \mathrm{s}$, более слабая, чем у героина. Данное различие является следствием разности в физико-химических свойствах веществ, а также из-за разности параметров гетерогенных процессов на поверхности эмиттера, происходящих при адсорбции молекул соответствующих веществ.
Данное различие можно видеть, также из спектров морфина (рис. 6) и по зависимости $T_{\max }$ от $T_{E}$ (рис. 7). Спектры морфина были получены при скорости $5^{\circ} \mathrm{C} / \mathrm{s}$. В случаях героина и папаверина, смещение закономерности $I_{s}(T)$ от $v_{i s}(T)$ и зависимость $T_{\max }$ от $T_{E}$ наблюдались только при $14.2^{\circ} \mathrm{C} / \mathrm{s}$, а при $7^{\circ} \mathrm{C} / \mathrm{s}$ не наблюдалось. В случае морфина скорости $5^{\circ} \mathrm{C} / \mathrm{s}$ было достаточно для возникновения данных эффектов.

Чем больше скорость развертки $\omega$, тем больше скорость изменения потока молекул $d v_{i s} / d T$, входящий к детектору. Когда $d v_{i s} / d T$ больше, быстродействие детектора может быть недостаточным. Смещение закономерности $I_{s}(T)$ от $v_{i s}(T)$ и зависимость $T_{\max }$ от $T_{E}$ являются следствием нехватки быстродействия детектора для того или иного вещества при данной скорости $d v_{i s} / d T(\omega)$.

Из ТДПИ спектров (рис. $2, b, 4, b$ и 6), а также из зависимостей $T_{\max }$ от $T_{E}$ (рис. $3, b, 5, b$ и 7) видно, что с увеличением $T_{E}$ эмиттера закономерность $I_{s}(T)$ приближается к закономерности $v_{i s}(T)$, а температуpa $T_{\max }$ спектра приближается к температуре $T_{i s \max }$. Это является следствием увеличения быстродействия детектора с увеличением $T_{E}$ эмиттера. А с некоторого значения $T_{E}$ эмиттера, быстродействие детектора примет значения, при которых закономерность $I_{s}(T)$ будет повторять $v_{i s}(T)$, а температура $T_{\max }$ будет равна $T_{i s \max }$. Дальнейшее увеличение $T_{E}$ не приводит к изменению закономерности $I_{s}(T)$ (за исключением интенсивности) и $T_{\max }$ спектров.

Нехватка быстродействия детектора для того или иного вещества при данной скорости $\omega$ и увеличение быстродействия детектора с увеличением $T_{E}$ можно объяснить на основе закономерностей нестационарных процессов ПИ. По этой причине была разработана математическая модель данной зависимости, основанная на ПИ молекул в нестационарных условиях.

На поверхность эмиттера адсорбируется $v_{a}=\eta v_{i s}$ часть потока испаряющихся молекул $v_{i s}(\eta-$ коэффициент использования веществ), значение которого изменяется со скоростью $d v_{i s} / d T$. В данном случае число молекул, находящихся в адсорбционном состояний $N$, число частиц продуктов химических реакций $N_{i}$ и молекул, десорбирующих в исходных состояниях $N_{M}$, а также суммарное число частиц $N_{s}$ изменяются со скоростью:

$$
\frac{d N}{d T}=\eta v_{i s}-\frac{A N}{\omega}, \quad \frac{d N_{i}}{d T}=\frac{\gamma_{i} A N}{\omega}-\frac{K_{i} N_{i}}{\omega}
$$

И

$$
\begin{gathered}
\frac{d N_{M}}{d T}=\frac{\gamma_{M} A N}{\omega}-\frac{K_{M} N_{M}}{\omega}, \\
\frac{d N_{s}}{d T}=\frac{A N}{\omega}-\frac{K_{M} N_{M}}{\omega}-\sum \frac{K_{i} N_{i}}{\omega},
\end{gathered}
$$

где $A=K_{M}+\sum_{z} K_{i}^{d}-$ скорость убыли молекул в адсорбированном состоянии; $z$ - число каналов химических реакций молекул; $\gamma_{i}\left(T_{E}\right)=K_{i}^{d} / A-$ коэффициент, определяющий долю адсорбированных молекул, которые вследствие подвергания гетерогенным химическим 

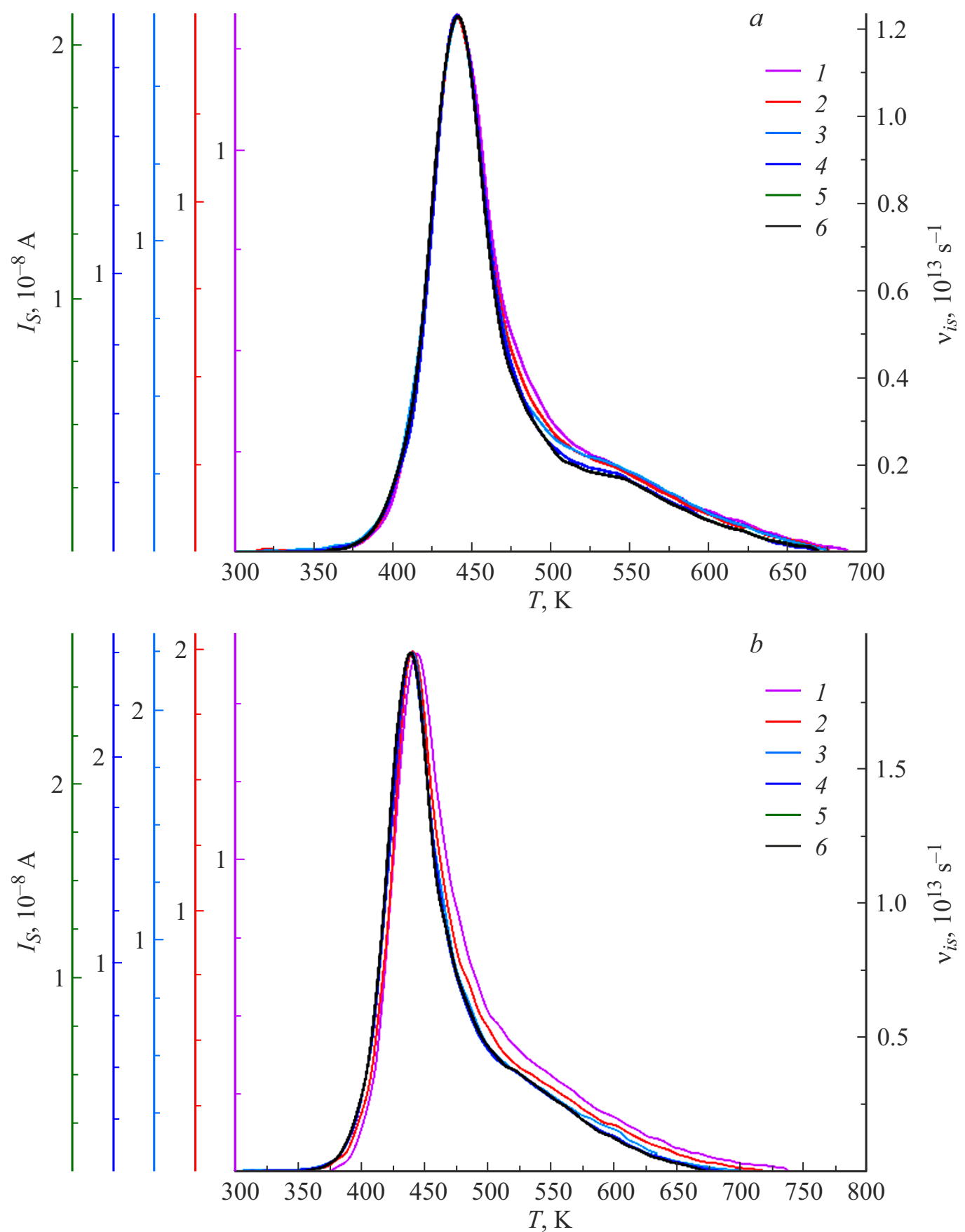

Рис. 4. ТДПИ спектры папаверина: $a-$ при $7 ; b-$ при $14.2^{\circ} \mathrm{C} / \mathrm{s}$. Значение $T_{E}, \mathrm{~K}: 1-639,2-649,3-659,4-668,5-$ 675,6 - поток молекул.

реакциям по $i$-му каналу преобразуются в частици $i$ го вида; $\gamma_{M}\left(T_{E}\right)=1-\sum_{z} \gamma_{i}\left(T_{E}\right)-$ коэффициент, определяющий долю адсорбированных молекул, которые десорбируются в исходном молекулярном состоянии; $K_{i}=K_{i}^{+}+K_{i}^{0}-$ суммарная скорость десорбции частиц $i$-го вида.

Поток частиц $i$-го вида и молекул, десорбированных с поверхности эмиттера, равны $v_{i}=K_{i} N_{i}$ и $v_{M}=K_{M} N_{M}$, а их сумма $v_{s}=\mu_{M}+\sum_{z} v_{i}$. Ток их ионов соответственно равен $I_{M}=e \beta_{M} v_{M}, I_{i}=e \beta_{i} v_{i}$, где $\beta_{M}$ и $\beta_{i}-$ коэффициенты ПИ частиц соответствующих видов. Регистрируемый суммарный ток равен:

$$
I_{s}=I_{M}+\sum_{z} I .
$$

Когда скорость изменения $d v_{i s} / d T$ потока адсорбирующихся молекул высокая, тогда скорость изменения числа частиц (1) и (2) также становится высокой. В этом случае суммарный поток десорбирующихся частиц $v_{s}$ 


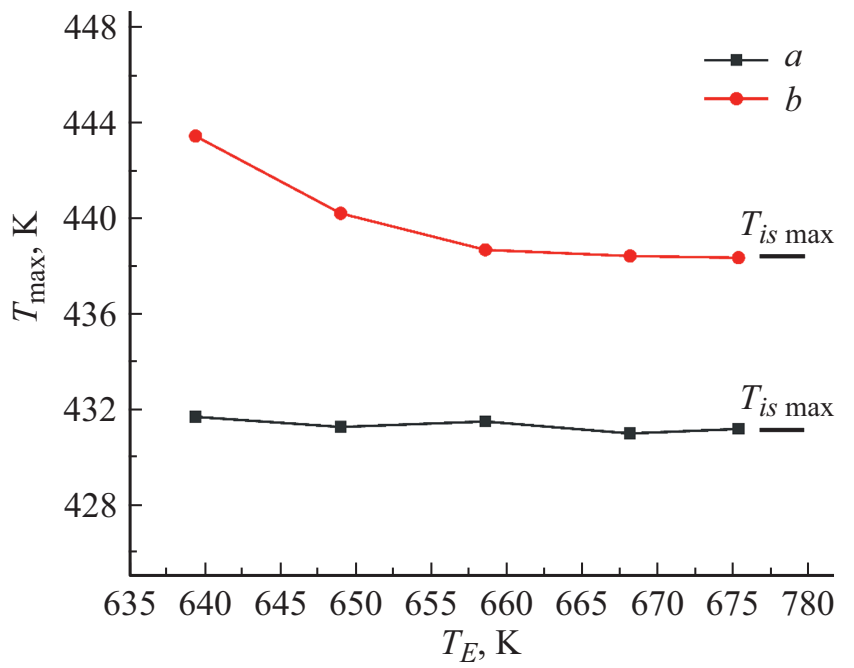

Рис. 5. Зависимость $T_{\max }$ от $T_{E}: a-$ при $7 ; b-$ при $14.2^{\circ} \mathrm{C} / \mathrm{s}$.

не будет равен потоку адсорбирующихся молекул $v_{a}$, т.е. $v_{s} \neq v_{a}$. Поэтому закономерность $I_{s}(T)$ не будет повторять закономерность $v_{i s}(T)$. А температура максимума спектра $T_{\max }$ не будет равна температуре максимума $T_{i s \max }$ потока молекул $v_{i s \max }$, поступающего к детектору. Данное состояние характеризуется как нехватка быстродействия детектора при данном $d v_{i s} / d T$. С увеличением температуры $T_{E}$ эмиттера скорость гетерогенных процессов $K_{M}, K_{i}^{d}, K_{i}$ увеличивается, приводя тем самым к уменьшению промежутка времени между адсорбцией молекул и десорбцией ионизируемых частиц. При таком случае значения (1) и (2) уменьшаются, закономерность $I_{s}(T)$ приближается к закономерности $v_{i s}(T)$, а значение $T_{\max }$ к $T_{i s \max }$. Данное состояние характеризуется как увеличение быстродействия детектора с увеличением $T_{E}$ эмиттера. С некоторого значения $T_{E}$ эмиттера значения $K_{M}, K_{i}^{d}, K_{i}$, становятся высокими. Промежуток времени между адсорбцией молекул и десорбцией ионизируемых частиц, а также значения (1) и (2) становятся незначительно малыми. Процессы, протекающие на адсорбционном слое эмиттера в таких условиях, можно считать квазистационарным. В этом случае закономерность $I_{s}(T)$ будет повторять закономерность $v_{i s}(T)$. Получится следующее равенство:

$$
v_{s}=\eta v_{i s}, \quad T_{\max }=T_{i s \max }=\mathrm{const}
$$

и

$$
I_{s}=e v_{i s} \eta\left(\gamma_{M} \beta_{M}+\sum_{z} \gamma_{i} \beta_{i}\right)=e E v_{i s},
$$

где $E$ - ионизационная эффективность веществ, определяемая экспериментальным путем. Данное состояние характеризуется как высокая степень быстродействия детектора при $d v_{i s} / d T$ и $T_{E}$ эмиттера.

\section{Заключение}

Таким образом, с увеличением температуры $T_{E}$ эмиттера увеличивается быстродействие детектора. Данная зависимость обусловлена тем, что с ростом температуры эмиттера увеличивается скорость гетерогенных процессов, протекающих в адсорбционном слое эмиттера. Увеличение скорости гетерогенных процессов приводит к уменьшению промежутка времени между адсорбцией

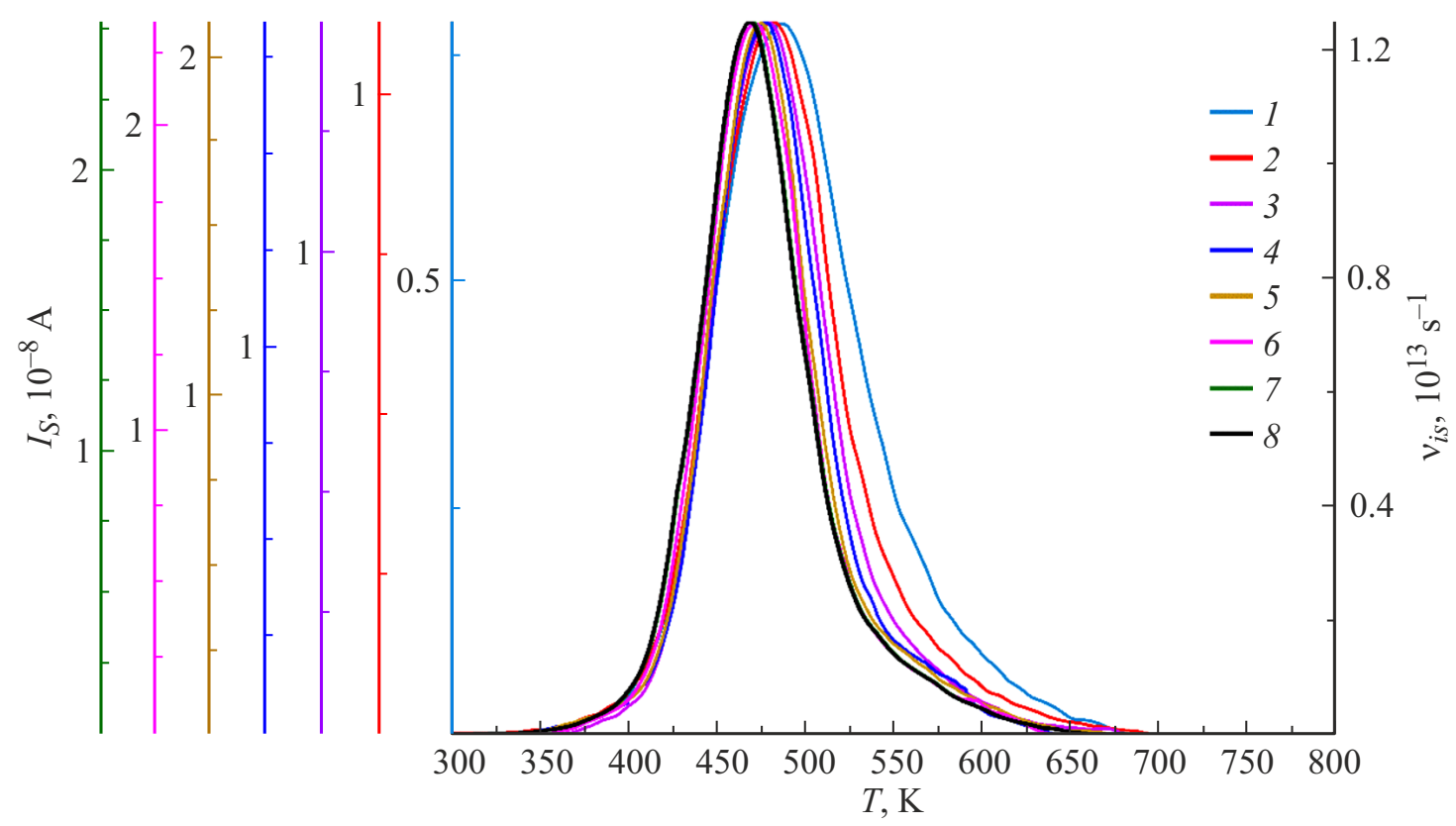

Рис. 6. ТДПИ спектры морфина. Скорость развертки $5^{\circ} \mathrm{C} / \mathrm{s}$. Значение $T_{E}, \mathrm{~K}: 1-635.4,2-645,3-655,4-668,5-683$, $6-696,7-708,8-$ поток молекул. 


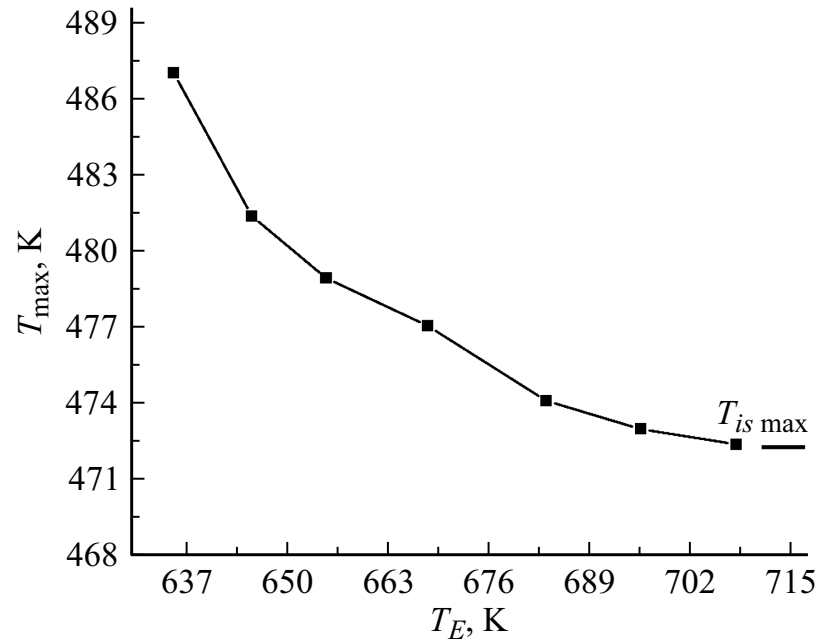

Рис. 7. Зависимость $T_{\max }$ от $T_{E}$.

молекул на поверхности эмиттера и десорбцией с нее ионизируемых частиц. Это приводит к уменьшению скорости изменения числа частиц в адсорбционном слое. В этом процессе закономерность спектра $I_{s}(T)$, получаемого детектором, приближается к закономерности спектра испарения веществ $v_{i s}(T)$. С некоторого значения $T_{E}$ эмиттера гетерогенные процессы, происходящие в адсорбционном слое, переходят в квазистационарное состояние. В этих условиях промежуток времени между адсорбцией и образованием ионов, а также скорость изменения числа частиц уменьшается до незначительного значения, при котором закономерность $I_{s}(T)$ будет повторять закономерность $v_{i s}(T)$, температура максимума спектра полученного детектором $T_{\max }$ будет равна температуре максимума спектра $T_{i s} \max$ испарения веществ. Дальнейшее увеличение $T_{E}$ эмиттера не приводит к изменению закономерности $I_{s}(T)$ и $T_{\max }$ спектра. Это приводит к тому, что задача нахождения режима работы детектора, при котором закономерность $I_{S}(T)$ будет повторять закономерность $v_{i s}(T)$ и $T_{\max }$ будет равна $T_{i s \max }$, переходит к задаче нахождения условия, при котором гетерогенные процессы в адсорбционном слое будут происходить в квазистационарных условиях.

Возникновение квазистационарных условий, помимо $T_{E}$ эмиттера, зависит также от скорости изменения потока адсорбируемых молекул $d v_{i s} / d T$. Когда скорость изменения $d v_{i s} / d T$ велика, скорость изменения числа частиц становится велика, и это в свою очередь приводит к смещению закономерности $I_{s}(T)$ от закономерности $v_{i s}(T)$. Тогда для возникновения квазистационарных условий требуется повышение $T_{E}$ эмиттера до более высоких температур.

Установление квазистационарных условий при определенных значениях $T_{E}$ и $d v_{i s} / d T$, зависит также от физико-химических свойств исследуемого вещества. При нагреве героина и папаверина со скоростью $7^{\circ} \mathrm{C} / \mathrm{s}$ в исследуемой области $T_{E}$ установлено квазистационарное состояние. Смещение закономерности $I_{S}(T)$ от закономерности $v_{i s}(T)$, вызванное не установлением квазистационарного условия, наблюдалось при скорости $14^{\circ} \mathrm{C} / \mathrm{s}$. А в случае морфина данное смещение наблюдается при $5^{\circ} \mathrm{C} / \mathrm{s}$.

\section{Финансирование работы}

Работа выполнена в рамках проекта МУ-Ф320171025268 и ФА-Атех-2018-17 Министерства инновационного развития Республики Узбекистан.

\section{Конфликт интересов}

Авторы заявляют, что у них нет конфликта интересов.

\section{Список литературы}

[1] J. Artiola, I.L. Pepper, M.L. Brusseau. Environmental Monitoring and Characterization (Elsevier Academic Press, USA, 2004)

[2] Е.А. Севрюкова. Мониторинг загрязнения окружсающей среды (Юрайт, М., 2016)

[3] Y. Wu, Y. Zhang. Food Chem. Toxicol., 56, 325 (2013). DOI: $10.1016 /$ j.fct.2013.02.044

[4] K. Vij. Textbook of Forensic Medicine and Toxicology. Principles and Practice (Elsevier, India, 2011)

[5] V.R. Preedy. Neuropathology of Drug Addictions and Substance Misuse. Vol. 2: Stimulants, Club and Dissociative Drugs, Hallucinogens, Steroids, Inhalants, and International Aspects (Elsevier Academic Press, USA, 2016)

[6] G.D. Christian, P.K. Dasgupta, K.A. Schug. Analytical Chemistry (John Wiley \& Sons, USA, 2013)

[7] N.A. Bakar, A. Abu-Siada, S. Islam. IEEE Electr. Insul. Mag., 30 (3), 39 (2014). DOI: 10.1109/MEI.2014.6804740

[8] J.R. Dean. Extraction Techniques in Analytical Sciences (John Wiley \& Sons, USA, 2009)

[9] R. Self. Extraction of Organic Analytes from Foods. A Manual of Methods (Royal Society of Chemistry, London, 2005)

[10] A.S. Franca, L.M.L. Nollet. Spectroscopic Methods in Food Analysis (Taylor \& Francis Group, London, 2018)

[11] B.M. Ham, A. MaHam. Analytical Chemistry: A Chemist and Laboratory Technician's Toolkit (John Wiley \& Sons, USA, 2016)

[12] U. Khasanov, U.Kh. Rasulev, D.T. Usmanov, S.S. Iskhakova. Proc. of the 11th Europ. Conf. on Applicat. Surf. Interface Anal., 26 (6), 309 (2006). DOI: 10.1002/sia.2257

[13] U.K. Rasulev, S.S. Iskhakova, U. Khasanov, A.V. Mikhalin. Int. J. Ion Mob. Spectrom., 4, 212 (2001).

[14] S.S. Iskhakova, A.V. Mikhailin, U.Kh. Rasulev, Ya.R. Sagatov, U. Khasanov. J. Anal. Chem., 59 (1), 50 (2004). DOI: 10.1023/B:JANC.0000011668.20677.d1

[15] С.С. Исхакова, У. Хасанов, У.Х. Расулев, Д.Т. Усманов. Письма в ЖТФ, 46 (24), 23 (2020). DOI: 10.21883/PJTF.2020.24.50423.18458 [S.S. Iskhakova, U. Khasanov, U.Kh. Rasulev, D.T. Usmanov, Tech. Phys Lett., 46 (12), 1231 (2020). DOI: 10.1134/S1063785020120196] 
[16] У.Х. Расулев, А.А. Балаухом, Б.Г. Второвым, Э.Г. Назаров, Г.Б. Худаева. Инфомационное сообшение АН УзССР, 470, 4 (1989).

[17] M. Holcapek, Wm.C. Byrdwell. Handbook of Advanced Chromatography / Mass Spectrometry Techniques (Elsevier Academic Press and AOCS Press, USA, 2017)

[18] J.H. Gross. Mass Spectrometry. A Textbook (Spring. Interntional Publish. AG, Switzerland, 2017)

[19] C.F. Poole. J. Chromatogr. A, 1421, 137 (2015). DOI: 10.1016/j.chroma.2015.02.061

[20] У.Х. Расулев, Э.Я. Зандберг, А.Г. Каменев, В.И. Полеев. ЖАХ, 35 (6), 1188 (1980).

[21] A. Hackner, A. Habauzit, G. Müller, E. Comini, G. Faglia, G. Sberveglieri. IEEE Sens. J., 9(12), 1727 (2009). DOI: 10.1109/JSEN.2009.2030705

[22] U.Kh. Rasulev, E.Ya. Zandberg. Prog. Surf. Sci., 28 (3-4), 181 (1988). DOI: 10.1016/0079-6816(88)90003-2 PROG SURF SCI

[23] K.E. Grafinger, W. Bernhard, W. Weinmann. Sci. Justice, 59 (4), 459 (2019). DOI: 10.1016/j.scijus.2019.03.005

[24] G. Pang. Analytical Methods for Food Safety by Mass Spectrometry: Volume I Pesticides (Elsevier Academic Press, USA, 2018)

[25] D.T. Usmanov, U. Khasanov, U.Kh. Rasulev. Chem. Nat. Compd., 39, 489 (2003). DOI: $10.1023 / \mathrm{B}: C O N C .0000011126 .68733 .19$

[26] U. Khasanov, U.Kh. Rasulev, D.T. Usmanov, S.S. Iskhakova. Proc. of the $11^{\text {th }}$ Europ. Conf. on Applicat. Surf. Interface Anal., 38 (4), 309 (2006). DOI: 10.1002/sia.2257

[27] U. Khasanov, U. Rasulev, D. Usmanov, S. Iskhakova, U. Nabiev. J. Pharmaceut. Biomed. 37 (5), 1125 (2005). DOI: 10.1016/j.jpba.2004.09.026

[28] D. Usmanov, U. Khasanov, A. Pantsirev, J. Bocxlaer. J. Pharmaceut. Biomed., 53 (4), 1058 (2010). DOI: $10.1016 /$ j.jpba.2010.07.004

[29] I. Ferrer, M.E. Thurman. Advanced Techniques in Gas Chromatography-Mass Spectrometry $(G C-M S-M S$ and GC-TOF-MS) for Environmental Chemistry, 61 (Elsevier, Great Britain, 2013)

[30] E. Lundanes, L. Reubsaet, T. Greibrokk. Chromatography, Basic Principles, Sample Preparations and Related Methods (Wiley-VCH Verlag GmbH \& Co. KGaA, Germany, 2014)

[31] F. Rouessac, A. Rouessac Chemical Analysis: Modern Instrumentation Methods and Techniques (John Wiley \& Sons, USA, 2013)

[32] Н.И. Царев, В.И. Царев, И.Б. Катраков. Практическая газовая хроматограбия (АГУ, Барнаул, 2000)

[33] К.А. Гольберт, М.С. Вигдергауз. Введение в газовую хроматографию (Химия, М., 1990)

[34] Е.С. Каратаев. Теоретические основы газовой хроматограбии (КНИТУ, Казань, 2015)

[35] Е.Е. Петушков, У.Х. Расулев, Э.Г. Назаров, А.А. Стамов, Ю.П. Козлов. Высокочистые вещества, 2, 127 (1993).

[36] У.Х. Расулев, Э.Г. Назаров, Г.Б. Худаева. Металлы, 5, 155 (1995).

[37] C. Cerquia, A. Ponzoni, D. Zappa, E. Comini, G. Sberveglieri. Procedia Engineer., 87, 1023 (2014). DOI: 10.1016/j.proeng.2014.11.335

[38] В.И. Капустин, В.С. Петров, А.А. Черноусов. Письма в ЖТФ, 30 (17), 19 (2004) [V.I. Kapustin, V.S. Petrov. Tech. Phys. Lett., 30 (9), 717 (2004). DOI: 10.1134/1.1804574]
[39] Д.В. Капустин, А.А. Буш, К.О. Нагорнов, В.И. Капустин. Письма в ЖТФ, $38(4), 83$ (2012). [D.V. Kapustin, A.A. Bush, K.O. Nagornov, V.I. Kapustin. Tech. Phys. Lett., 38 (2), 197 (2012). DOI: 10.1134/S1063785012020277]

[40] D.T. Usmanov, U. Khasanov. J. Surf. Investig-X-Ray, 5, 503 (2011). DOI: 10.1134/S1027451011020194

[41] D.T. Usmanov, Sh.Dj. Akhunov, U. Khasanov, V.M. Rotshteyn, B.Sh. Kasimov. Eur. J. Mass Spectrom., 26 (2), 153 (2020). DOI: $10.1177 / 1469066719875655$

[42] Sh. Akhunov, Kh. Ashurov, Sh. Axmedov, B. Kasimov, V. Rotshteyn, A. Radjabov, D. Usmanov. Eur. J. Mass Spectrom., 27 (1), 29 (2021). DOI: $10.1177 / 14690667211002777$

[43] S. Takahashi, F. Nagamura, M. Sasaki, T. Fujii. Chem. Pap., 63 (5), 613 (2009). DOI: 10.2478/s11696-009-0062-2

[44] W. Li, D. Wu, Sh. Chen, H. Peng, Y. Guan. J. Chrom. A, 1218, 6812 (2011). DOI: 10.1016/j.chroma.2011.07.074

[45] A. Hackner, W. Legner, G. Müller, E. Biavardi, E. Dalcanale, S. Zampolli, I. Elmi, G. Carlo Cardinali. Sens. Actuat. B, 185, 771 (2013). DOI: 10.1016/j.snb.2012.09.030

[46] W. Li, Y. Guan, Z. Shena, D. Wu. Chem. Commun., 47, 2423 (2011). DOI: 10.1039/C0CC04528D

[47] G. Müller, J.D. Prades, A. Hackner, A. Ponzoni, E. Comini, G. Sberveglieri. Nanomaterials, 8(1017), 22 (2018). DOI: $10.3390 /$ nano8121017

[48] Э.Г. Назаров, У.Х. Расулев. Нестащионарные процессы поверхностной ионизации (Фан, Ташкент, 1991)

[49] A. Kholov, K.S. Tursunov, U.Kh. Rasulev, E.G. Nazarov, M.N. Lapushkin, M.V. Knat'ko, E.Ya. Zandberg, I.L. Zhuravleva. Teor. Eksp. Khim, 20 (4), 405 (1984). DOI: $10.1007 / \mathrm{BF} 00516575$

[50] Э.Я. Занберг, Н.И. Ионов, У.Х. Расулев, Ш.М. Халиков. ЖТФ, 47, 133 (1978).

[51] Э.Я. Зандбер, Э.Г. Назаров, У.Х. Расулев. ЖТФ, 50, 1752 (1980).

[52] Э.Я. Зандбер, Э.Г. Назаров, У.Х. Расулев. ЖТФ, 51, 123 (1981).

[53] G. Rakhmanov, U.Kh. Rasulev, I. Saidumarov. Proc. of the 11th Europ. Conf. Appl. Surf. Interface Anal., 38(4), 219 (2006). DOI: 10.1002/sia.2206 